Revue de l'Institut des langues et cultures

d'Europe, Amérique, Afrique, Asie et Australie

23 | 2015

Le national-socialisme dans son cinéma

\title{
La postérité stylistique du Triomphe de la volonté et des Dieux du stade
}

The Stylistic Posterity of Leni Riefenstahl's Triumph of the Will and Olympia

\author{
Laurent Jullier
}

\section{OpenEdition}

Journals

Édition électronique

URL : http://journals.openedition.org/ilcea/3357

DOI : 10.4000/ilcea.3357

ISSN : 2101-0609

\section{Éditeur}

UGA Éditions/Université Grenoble Alpes

\section{Édition imprimée}

ISBN : 978-2-84310-305-6

ISSN : $1639-6073$

Référence électronique

Laurent Jullier, « La postérité stylistique du Triomphe de la volonté et des Dieux du stade », ILCEA [En ligne], 23 | 2015, mis en ligne le 09 juillet 2015, consulté le 01 mai 2019. URL : http:// journals.openedition.org/ilcea/3357 ; DOI : 10.4000/ilcea.3357

Ce document a été généré automatiquement le 1 mai 2019.

(C) ILCEA 


\title{
La postérité stylistique du Triomphe de la volonté et des Dieux du stade
}

\author{
The Stylistic Posterity of Leni Riefenstahl's Triumph of the Will and Olympia
}

\author{
Laurent Jullier
}

Dans le champ de l'histoire des styles cinématographiques, une assertion courante consiste à voir la manière de filmer de Leni Riefenstahl, au moins telle que la postérité l'a fixée dans Le Triomphe de la volonté et Les Dieux du stade, comme l'annonce ou la préfiguration du style postmoderne ${ }^{1}$. Cette affirmation d'une "contemporanéité rétrospective" pose au moins deux problèmes épistémologiques, l'un concernant l'histoire de l'art et l'autre la culture visuelle: (1) les inventions stylistiques de Leni Riefenstahl sont-elles des trouvailles ex nihilo ou des emprunts aux figures du Zeitgeist de son époque? (2) une figure de style qui est techniquement la même, à cinquante ans de distance, reste-t-elle cognitivement la même?

Avant d'y réfléchir, quelques précautions d'ordre éthique s'imposent. Décontextualiser des films sulfureux afin de se concentrer sur leurs propriétés formelles, c'est-à-dire passer "de la transparence à l'opacité ${ }^{2}$ " dans un geste typique de ce qu'est devenue l'esthétique kantienne appliquée aux arts plastiques, ne va pas sans poser quelques problèmes de conscience. Ces films sont peut-être des réussites formelles, mais ils s'inscrivent dans une logique de pensée politique qui a abouti aux horreurs de la Seconde Guerre mondiale. La question n'est pas de savoir si à l'époque ils ont servi d'armes de propagande: quand Brian Winston (1997) dit qu'ils sont bien trop formalistes pour convaincre quiconque de prendre sa carte du parti nazi (alors qu'à l'inverse, Le Cuirassé Potemkine donnerait envie à n'importe qui de voter communiste), il joue sur les mots. Sans doute ne constituent-ils pas de la propagande directe, mais personne ne niera qu'ils peuvent servir à véhiculer l'image d'un régime politique «normal », qui montre assez de goût pour subventionner les belles images et, apparemment, n'enferme ni n'extermine personne pour délit d'opinion, de religion ou de préférence sexuelle. La question est plutôt de savoir si poser sur eux un regard formaliste les réhabilite tout entiers, comme si ce regard acquérait une valeur rédemptrice et un pouvoir performatif. 
On ne cherchera pas, dans les limites de cet article, la réponse à cette question dans les propos de Leni Riefenstahl, qui a passé les deux derniers tiers de sa longue vie à expliquer qu'elle n'était pour rien dans les dérives du régime nazi, et qu'elle s'était contentée, plus jeune, de faire les meilleures images possibles. Sa défense a été parnassienne, pourrait-on dire : elle mettait moins l'accent sur la qualité propre des formes qu'elle avait mises dans ses films que sur la somme de travail acharné qui avait été nécessaire à leur lente élaboration, incluant le bricolage inventif de toutes sortes de machines et les négociations de toutes sortes de permissions avec les autorités concernées par les événements filmés. Mais elle n'a jamais triomphé de ces problèmes éthiques, qui d'ailleurs la poursuivent post-mortem : l'exposition d'art allemand que le Louvre a récemment organisée mettait les Dieux du stade en position d'exemplifier le Troisième Reich ${ }^{3}$. Et il suffit de regarder les user reviews (commentaires des internautes) de l'IMDb, la plus grande base de données cinéma du web, pour s'apercevoir de son échec en la matière ${ }^{4}$.

Susan Sontag a en partie retracé en $1975^{5}$ la façon dont le «mythe Riefenstahl », visant à ériger la cinéaste en héraut de la cause formaliste du "progrès en art " en faisant abstraction de tout lien avec le nazisme - un peu sur le modèle de ce qui s'est passé avec les sympathies de David W. Griffith pour le Ku Klux Klan -, a été construit au fil des années par un certain nombre d'intellectuels et d'artistes américains et français d'obédience moderniste. Elle n'avait que l'embarras du choix, de Jean Cocteau à « Jonas Mekas qui célébrait son idéalisme en disant qu'il fallait être nazi pour voir une apologie du nazisme dans ses films ${ }^{6} "$, en passant par des ouvrages universitaires comme celui de Richard Barsam (1975), et l'entrevue avec les Cahiers du Cinéma en septembre 1965, où Riefenstahl se réclamait du " cinéma-vérité » cependant que son interlocuteur expliquait que Les Dieux du stade et le Triomphe construisaient « une réalité basée sur une certaine idée de la forme » (Delahaye, 1965). Et si l'article de Sontag date de 1975, on se convaincra que rien n'a changé par la suite en lisant ceux que François Albera a écrit plus récemment pour épingler lui aussi ce regard formaliste biaisé, qui affleure par exemple sous les plumes de Jean-Michel Frodon ou de Bernard Eisenschitz ${ }^{7}$. La longévité de la cinéaste n’a fait que renforcer ce mythe en effaçant un peu plus les traces de ses liens avec le régime nazi : le jour de ses cent ans, le 22 août 2002, les journaux français bombardaient leurs lecteurs des «expressions stéréotypées de circonstance, la toute nouvelle centenaire étant présentée comme une éternelle jeune fille, une figure mythique, une légende vivante, une aventurière fabuleuse » (Richard, 2002).

Les allusions que Quentin Tarantino a faites dans son film Inglourious Basterds, en 2009, vont, pour finir, également dans le même sens. On y voit Shoshanna Dreyfus, l'hérö̈ne, répondre à un soldat allemand qui s'étonne de la voir programmer dans sa salle de cinéma parisienne un film dont la vedette est Leni Riefenstahl ${ }^{8}$ :

- Je suis française ; ici nous respectons les réalisateurs.

6 Tarantino, face aux médias qui lui reprochent son utilisation de sa violence, adopte d'ailleurs un système de défense formaliste comparable à celui de Riefenstahl : ce ne sont que des images, ne confondez pas ce qui se passe sur l'écran avec ce qui se passe dans la vraie vie. Pour le spectateur qui vient de voir leurs films, c'est-à-dire de se faire simplement raconter une histoire qui obéit à certaines conventions génériques, il n'y aurait donc pas plus de raisons de se lancer dans la justice personnelle létale chère à la plupart des héros tarantiniens que d'exterminer des populations entières par sympathie avec la cause nazie. 
7 À défaut de revenir dans ce débat complexe sur les rapports qu'entretiennent les images et le monde, peut-on au moins régler la question stylistique ? Aux yeux d'un historien du cinéma comme François Albera, "on cherche encore et toujours à construire la "génialité" de Leni Riefenstahl, échappant au poids de son époque. La connaissance du contexte tant artistique (la danse) que sportif (les Jeux) permet pourtant de rompre avec la mythologie de l'esthétique riefenstahlienne » (2003). Présentons donc, pour essayer de répondre aux questions posées plus haut, quelques connaissances relatives à ce contexte artistique.

Pour commencer, nous avons affaire à une hybridation: une part du style de Leni Riefenstahl relève d'un académisme désormais démodé, et une autre part se lit aisément comme l'écho des expérimentations des années 1920, dont certaines ont désormais intégré le vocabulaire visuel dominant dans les arts et les médias.

Son académisme s'observe essentiellement à deux endroits: le goût pour la contreplongée et le respect de la règle des trois tiers. Le premier trouve son origine dans l'utilisation de piédestaux en sculpture, qui obligent à lever la tête pour voir les figures représentées en jouant sur l'association entre ce qui est haut et ce qui est fort, ou puissant, ou divin (dans le cas des religions qui placent les dieux au ciel). Dans la même logique intermédiale, la règle des trois tiers, elle, est passée de la peinture classique aux manuels pour photographes puis cinéastes débutants (elle s'y trouve encore de nos jours), qui suppose d'inscrire le sujet ou les lignes de partage des surfaces le long des deux horizontales et des deux verticales qui coupent le cadre en trois parties égales. Du côté des solutions formelles plus osées, disons d'emblée que tout ce que l'on peut voir dans les deux films de Leni Riefenstahl dont il est question ici peut s'observer ailleurs, notamment dans les films dadaïstes, futuristes et chez les cinéastes soviétiques néoformalistes, ainsi que - pour citer des sources auxquelles elle a plus sûrement été exposée - chez les cinéastes allemands de l'expressionnisme et de l'Entfesselte Kamera (la caméra libérée de ses chaînes). Il s'agit principalement du ralenti, de l'inversion du sens de défilement, du match cut et des mouvements de caméra immersifs.

Commençons par le ralenti, devenu très courant, notamment dans des formats postmodernes comme le blockbuster d'action, la publicité, le clip, les retransmissions sportives et les jeux vidéo en mode replay (notamment les jeux de sport). Leni Riefenstahl utilise amplement le ralenti dans les Dieux du stade, associé parfois à l'inversion du sens de défilement (figure qu'affectionnait Cocteau). Cependant, elle n'est pas la seule. Les deux Bergfilms d'Arnold Fanck desquels elle est la vedette féminine, Der Heilige Berg (1926) et Der Weisse Rausch. Neue Wunder des Schneeschuhs (1931), en contiennent déjà, notamment dans des scènes mettant en scène de talentueux skieurs. L'association du ralenti et du sport, héritage du cinéma scientifique et même de la chronophotographie ${ }^{9}$, se trouve aussi dans Taris roi de l'eau (Jean Vigo, 1931). Mais le ralenti, à cette époque, est aussi employé à d'autres desseins: les cinéastes impressionnistes français l'utilisent à des fins de défamiliarisation romantique, comme Jean Epstein dans La Chute de la maison Usher en 1928, et les cinéastes dada pour sa capacité à rendre dérisoires et un peu ridicules les mouvements du corps humain, comme René Clair dans Entracte en 1924. Dans les Dieux du stade, cependant, Leni Riefenstahl utilise le ralenti pour magnifier et fluidifier les mouvements ; or parmi toutes les fonctions du ralenti présentes à son époque, c'est celleci qui domine de nos jours au cinéma - cette jouissance de profiter plus longtemps d'un spectacle qui d'ordinaire se dérobe au regard. 
11 Cette vista artistique de la cinéaste va se répéter dans le cas des autres figures. Le match cut, qui consiste à relier deux plans sur la base d'une ressemblance plastique ou sémantique entre un objet présent à la fin du plan A et un autre objet présent au début du plan B, se trouve lui aussi dans le Kunstwollen cinématographique de son temps - Fanck en place plusieurs dans Der Heilige Berg (analogie entre les pistils d'une fleur de pissenlit en fin de plan A et les flocons de neige en début de plan B), et on peut en voir quantité dans Menschen am Sonntag (Robert Siodmak \& Edgar G. Ulmer, 1930). Par exemple, dans le Triomphe, deux jeunes femmes qui attendent l'arrivée d'Adolf Hitler se passent la langue sur les lèvres, l'une en fin de plan A, l'autre en début de plan B. Cette figure était prohibée dans le style classique hollywoodien en dehors de quelques effets comiques (comme dans The Strawberry Blonde, Raoul Walsh, 1941), car elle attire l'attention sur l'aspect plastique au détriment de l'absorption dans la diégèse ${ }^{10}$. Leni Riefenstahl a d'ailleurs déclaré dans un des documentaires télévisés qui lui sont consacrés, Die Macht der Bilder: Leni Riefenstahl ${ }^{11}$ qu'à cause de cette organisation du montage par une logique plastique, le Triomphe « ressemble plus à un film d'art qu'à un documentaire ». Or le match cut, surtout si on le considère au sens large comme un raccord-mouvement sans raccord-objet, est désormais une figure dominante dans les spots publicitaires et surtout les vidéoclips.

Mais ce qui rend Le Triomphe de la volonté et Les Dieux du stade si familiers à l'œil d'un jeune spectateur d'aujourd'hui est sans doute, encore plus que le ralenti et le match cut, la façon qu'a leur réalisatrice d'utiliser les mouvements de caméra immersifs. D’innombrables photos et making of, comme on ne disait pas encore, la montrent faisant placer des caméras dans des endroits invraisemblables - ballons, trous, câbles tendus - ou juchée la tête à l'œilleton sur des chariots que des assistants poussent en tous sens. Là non plus il ne s'agit pas d'une innovation: Abel Gance et F. W. Murnau ont bien avant elle fait construire des machines plus folles et fait exécuter des mouvements plus virtuoses. Mais Gance et Murnau agissaient de cette façon pour se mettre au service de l'histoire fictionnelle qu'ils racontaient; leurs mouvements étaient chargés de déployer des métaphores (Gance) ou de décrire par un biais kinesthésique les sentiments intimes des personnages (Murnau). Tandis que dans Le Triomphe de la volonté et Les Dieux du stade, ils ne répondent qu'à des problèmes plastiques. Ces problèmes sont de trois ordres, selon que (1) l'objet à filmer ne bouge pas, (2) il bouge lentement, (3) il bouge vite. Les deux films qui nous occupent montrent les solutions trouvées pour chacun de ces trois cas :

1. Mimant le geste du badaud qui tourne autour de la statue juchée sur son socle, des travellings circulaires nous font tourner autour des athlètes sculptés néo-classiques d'Arno Breker (Les Dieux ${ }^{12}$ ) et autour de Hitler discourant (Le Triomphe), chaque fois en contreplongée.

2. Pour dynamiser des plans qui risqueraient d'ennuyer le spectateur, Leni Riefenstahl choisit parfois de filmer des objets qui se déplacent lentement de façon perpendiculaire à leur déplacement. C'est ce qui arrive dans Le Triomphe quand la caméra placée dans un montecharge grimpe en travelling ascendant cependant que Heinrich Himmler et Viktor Lutze, 
pour aller rendre hommage aux victimes allemandes de la Première Guerre mondiale, marchent entre les rangs des sections aux milliers de membres droits comme des piquets.

3. Pour suivre les coureurs sur le stade olympique, une caméra tirée file le long d'un câble à la même vitesse qu'eux, comme le lapin mécanique dans les courses de lévriers.

l'ordinaire des écrans du monde :

1. Basés sur le contraste entre ce qui bouge (l'observant) et ce qui ne bouge pas (l'observé), les travellings circulaires autour de personnes statiques appartiennent au vocabulaire de la télévision et à celui de cinéastes qui annoncent le style postmoderne dès la fin des années 1960, comme Claude Lelouch ou Brian de Palma. De nos jours, les réalisateurs de superproduction épiques comme Peter Jackson ou Michael Bay en placent volontiers sous forme aérienne dans les scènes d'extérieur ${ }^{13}$. Les retransmissions sportives commencent même, désormais, à combiner ralenti et travelling circulaire à l'occasion d'《 effets-Matrix ${ }^{14}$ ».

2. Le choix de filmer les acteurs et les objets mobiles dans un sens perpendiculaire à leur déplacement - sinon parfois opposé à leur déplacement - est désormais un standard du cinéma d'action. Parmi les internautes de l'IMDb qui ont laissé un commentaire sur le Triomphe, l'un a d'ailleurs remarqué que le travelling latéral qui suit le plan sur Himmler et Lutze « a été repris par George Lucas à la fin du premier Star Wars, en $1977^{15}$ ».

3. Le bricolage de Leni Riefenstahl destiné à suivre les coureurs sur le stade olympique est aujourd'hui un passage obligé des retransmissions sportives, par exemple avec le Speedtrack ${ }^{16}, \mathrm{l}^{\prime}$ AirTrack $\mathrm{Cam}^{17}$ et les systèmes de steadicam montées sur voiturettes.

Dlus, Leni Riefenstahl a aussi montré du goût pour les travellings avant immersifs sans doute le mouvement le plus courant du cinéma d'action et des jeux vidéo actuels, par exemple dans les ouvertures du Triomphe (en avion) et de Tag der Freiheit-Unsere Wehrmacht (1935). Même si là encore elle est loin d'avoir inventé ce procédé, Der Weisse Rausch contenant déjà des travellings avant sur luge et Menschen am Sonntag des travellings avant en voiture, sans parler du fait que les Lumière l'avaient inauguré dès 1896, ses choix continuent d'être rétrospectivement « justes». Mentionnons aussi, parmi les figures désormais familières et banales, le long travelling arrière en voiture sur la foule en liesse à la fin du Triomphe, passage obligé des concerts rock dans les stades, et désormais exécuté à la Louma ${ }^{18}$ de manière à faire éprouver la pure euphorie du nombre et de la communauté d'élan, ainsi que les quelques plans " subjectifs ${ }^{19}$ des marathoniens qui courent dans Les Dieux, qui seraient facilement incluables désormais dans une séquence tournée en run and gun ${ }^{20}$.

Une illustration de la « contemporanéité rétrospective » du style de Leni Riefenstahl peut être fournie par le clip du groupe de metal Rammstein intitulé Stripped (Phillip Stölzl \& Sven Budelmann, 1998). Cependant que les musiciens exécutent leur «musique métronomique aux accents militaires, accompagnée d'un chant à la fois rauque et sévère » (Mombelet \& Walzer, 2007 : 293), les réalisateurs se sont contentés de remonter quelques extraits des Dieux du stade. Et bien sûr ces extraits " collent " parfaitement, en toute synesthésie - le résultat aurait d'ailleurs été tout aussi fluide avec d'autres styles de musique, techno, rap, etc., pourvu que la manière de monter les plans sur le rythme de la musique ne changeât pas. Les paroles de Stripped, en effet, ne font aucunement référence au nazisme, pas plus que les membres de Rammstein ni leurs fans ne se réclament de cette idéologie ; ce sont de simples allusions. À quoi bon les faire, dira-t-on? Dans la réponse à cette question, on va voir que la «justesse " rétrospective des choix esthétiques de Leni Riefenstahl se double d'une adéquation avec le rapport 
qu'entretiennent nombre de citoyens postmodernes aux idées et pratiques " extrêmes ». Les métalleux (fans de musique metal)

[...] reconnaissent volontiers l'aspect paradoxal de leur démarche, [qui consiste à] afficher un symbole évoquant le régime nazi sans signifier qu'ils adhèrent à l'idéologie correspondante, mais ils sont séduits par la musique et l'aura subversive qu'elle dégage [...]. En règle générale, mus par une recherche de puissance et non de pouvoir, les métalleux jouent avec les interdits sociaux. (Mombelet \& Walzer, 2007 : 304, 307)

Choquer autrui - ici, en faisant référence au régime nazi, ne va pas, en effet, sans contrepartie narcissique : «Il est jouissif, pour eux, de savoir qu'ils ont la mainmise sur les émotions et les ressentis d'autrui. » (Mombelet \& Walzer, 2007 : 297) Encore une façon - politique et non plus esthétique, cette fois - pour les films de Riefenstahl d'être bien dans l'air du temps.

Il est maintenant possible de d'apporter des éléments de réponse aux questions de départ. Les inventions stylistiques de Leni Riefenstahl dans les deux films considérés ne sont pas, $\mathrm{du}$ point de vue technologique, des trouvailles ex nihilo mais des emprunts aux figures du Kunstwollen de son époque. Cependant, le fait qu'elle combine ces figures entre elles (principalement le match cut, le ralenti et les mouvements de caméra immersifs), ainsi que l'usage systématique et volontiers "gratuit » qu'elle fait d'elles (au sens où cet usage n'obéit pas de façon néoformaliste à un souci de coller aux exigences du profilmique ni à celles de la diégèse ${ }^{21}$ ), font d'elle une bonne candidate au titre de précurseur du style postmoderne. Ainsi sa façon de sacrifier la puissance narrative d'un geste technique à l'effet quasi-hypnotique qu'il entraîne chez le spectateur se retrouve-t-elle chaque jour chez les réalisateurs de clips.

17 Cette «hypothèse $d u$ précurseur» souffre cependant d'un gros problème épistémologique. Elle est en effet de nature postdictive. Le terme de postdiction, en psychologie, indique la propension de notre système perceptivo-cognitif à tenir paradoxalement compte, lorsqu'il s'agit de construire l'idée que l'on se fait d'un événement, d'informations postérieures à l'occurrence de cet événement. Par extension, on peut qualifier ainsi les lectures hégéliennes de l'histoire de l'art qui construisent des filiations dans le sens de la "naissance» d'un trait artistique en direction de sa «maturité » puis de son " déclin », et constituent en fait des vectorisations quelque peu abusives, sauf dans une perspective réductionniste d'autonomisation du " texte visuel». En effet, les figures de style ne naissent pas comme les êtres vivants, et revenir en arrière pour qualifier de "naissance » quelque chose qui ne peut être vu comme tel qu'avec le recul du temps et la volonté d'établir une descendance n'a de la validité que dans le cadre méthodologique d'une certaine histoire de l'art isolant le style de tout contexte de production et de réception. Pour le dire autrement, il est difficile de soutenir qu'une figure de style qui est techniquement la même, à cinquante ans de distance, reste cognitivement la même. Lorsqu'un spectateur des années 1930 regarde un match cut, un ralenti ou un travelling circulaire, il perçoit la même chose qu'un spectateur des années 2010 qui les regarde, car la perception est régulée par des automatismes encapsulés insensibles à la culture; en revanche il ne voit pas la même chose au sens d'appréhender, comprendre ou goûter le spectacle, car la cognition est régulée par quantité de scripts où s'imbriquent la culture personnelle et la mémoire épisodique. Or l'histoire a suivi son cours. Au long des quatre-vingts ans qui séparent ces deux spectateurs, les écrans du monde se sont multipliés hors des salles de cinéma pour envahir le quotidien, et se sont trouvés peu à peu infestés de match cuts, de ralentis et de 
travellings en tous sens ${ }^{22}$. Ce qui était exceptionnel est devenu la norme. Seule une lecture anhistorique, insensible à la notion de culture visuelle, peut donc faire de Leni Riefenstahl une figure d'inventeur ou de précurseur.

\section{BIBLIOGRAPHIE}

ALBERA François (2003), « Les 100 ans de Léni Riefenstahl : retour du même », 1895, 40, en ligne sur $<$ http://1895.revues.org/3512>.

ALBERA François (2008), « Leni Riefenstahl dans le Journal de Joseph Goebbels (1929-1944) », 1895, 55, en ligne sur <http://1895.revues.org/4108>.

BARSAM Richard M. (1975), Film Guide to Triumph of the Will, Bloomington : Indiana University Press.

DAGEN Philippe \& LEMAîTRE Frédéric (2013), « De l'Allemagne : le grand malentendu », Le Monde, 18 avril 2013.

Delahaye Michel (1965), «Leni et le loup : entretien avec Leni Riefenstahl », Cahiers du Cinéma, 170, 42-63.

JULLIER Laurent (1997), L'Écran post-moderne, Paris : L'Harmattan.

JULLIER Laurent (2012), « Une rétro-ingénierie du regard. L'exemple des voyages de Scrooge », J. Nacache \& J.-L. Bourget (dir.), Cinématismes. La littérature au prisme du cinéma (73-90), Berne : Peter Lang.

JULLIER Laurent (2013), « Dis-moi ce que tu vois ! Le régime visuel du run and gun », Mise au Point, 5 (Le cinéma européen et les langues), en ligne sur <https://map.revues.org/1371>. JunOD Philippe (1976), Transparence et opacité. Essai sur les fondements théoriques de l'art moderne, Lausanne : L'Âge d'Homme.

Mombelet Alexis \& Walzer Nicolas (2007), « Musique metal, idéologies extrémistes et rapport au politique ", J.-M. Seca, Musiques populaires underground et représentations du politique (290-310), Fernelmont : E.M.E. \& InterCommunications.

RICHARD Lionel (2002), «Indécente réhabilitation de Leni Riefenstahl », Le Monde diplomatique, octobre 2002, 36.

SONTAG Susan (1980), « Fascinating Fascism », Under the Sign of Saturn (73-105), New York : Farrar, Straus \& Giroux.

Winston Brian (1997), « Triumph of the Will », History Today, 47(1), 24-28.

\section{NOTES}

1. J'y ai souscrit quelque peu imprudemment il y a quelques années, sans les précautions dont il est question dans cet article : voir Jullier (1997 : 76). 
2. Celle du style, s'entend : voir Junod (1976).

3. «De L'Allemagne : 1800-1939, de Friedrich à Beckmann », Le Louvre, printemps 2013. Des voix se sont élevées outre-Rhin pour y contester la présence de «Leni Riefenstahl, cinéaste préférée d'Hitler et auteure du Triomphe de la volonté, à la gloire du Führer» (Dagen \& Lemaître, 2013).

4. Sur la centaine de user reviews du Triomphe, les mises en garde dominent largement. Par exemple : «Quiconque parle d'équivalence entre vérité et beauté devrait regarder ce film. Il est certainement très beau, mais il est aussi au service d'un mensonge, et l'un des pires » (<CTS-1>, 2005) ; « un film-oxymore : chef-d'œuvre nazi » (<sychonic>, 2000); «Comment pouvez-vous regarder une chose pareille? Comment est-il possible que ça se vende en DVD ? C'est une insulte envers les millions de personnes mortes à cause de l'holocauste et de la guerre que ce régime avait déclenchée " (<tarantinoarchives>, 2002). Il y a moins de $10 \%$ de lectures formalistes du type: "Laissez vos jugements de côté et appréciez l'expertise technique mise dans cette captation d'un Woodstock nazi » (<HAL-98>, 2001). En ligne sur <www.imdb.com/title/tt0025913/ reviews>

5. Sontag (1980). Certaines idées présentes dans ce papier (paru à l'origine dans la New York Review of Books du 6 février 1975 et lisible en ligne sur <www.nybooks.com/articles/ archives/1975/feb/06/fascinating-fascism/>) sont bien plus discutables, mais ce n'est pas le lieu ici d'en discuter car ils concernent les autres films de Leni Riefenstahl.

6. Sontag cite ici des papiers de Mekas parus dans les numéros du Village Voice des 31 octobre et 7 novembre 1974.

7. Voir Albera (2003): <http://1895.revues.org/3512>; Albera (2008): < http://1895.revues.org/4108>.

8. L’Enfer blanc du Piz Palü (Die weiße Hölle vom Piz Palü, Arnold Fanck \& Georg Wilhelm Pabst, 1929). 9. Les retransmissions sportives donnent désormais à voir des plans tournés à la Superloupe ${ }^{\mathrm{TM}}$, une caméra qui permet de filmer jusqu'à 7000 images/seconde (voir <www.dvs-sport.tv/ sousrubrique.php?rub=2>), ce qui est le score qu'atteignait dans les années 1910 Lucien Bull, pionnier du cinéma ultrarapide et disciple de l'inventeur de la chronophotographie Étienne-Jules Marey.

10. On pourrait en dire autant de la «cathédrale de lumière " conçue par Albert Speer pour l'éclairage de la Zeppelintribune du congrès de Nüremberg immortalisé par le Triomphe: elle était composée de 130 projecteurs de défense anti-aérienne, mais les pinceaux lumineux perdaient leur fonction guerrière première en étant disposés « harmonieusement ».

11. Réalisé en 1993 par Ray Müller. Celui-ci, à la fois fasciné et horrifié par son personnage, reproduit dès l'ouverture de son téléfilm l'un des mouvements de caméra immersifs « inutiles » qui lui étaient chers à l'époque nazie... sans qu'on sache si c'est un hommage ou un réemploi ingénu.

12. Des plans quasi-identiques se trouvent dans Le Mépris (Jean-Luc Godard, 1963).

13. Ils sont tournés à l'aide d'hélicoptères télécommandés, et désormais de drones : voir < www.etalors.com/drone>.

14. Scission du temps de l'action et du temps d'exploration visuelle de cette action; voir pour le sport <www.replay-technologies.com>.

15. <Ed Uyeshima $>$ (2006), User reviews du Triomphe, en ligne sur <www.imdb.com/title/ tt0025913/reviews>.

16. $<$ www.aerial-france.fr/modules/means_detail.asp?id=22>.

17. <www.airtrackcam.com>.

18. Caméra montée sur grue à bras télescopique ; voir <www.loumasystems.biz>

19. Comme la plupart des plans de coupe des Dieux, ils ont été tournés hors de la période des Jeux pour ne pas gêner la compétition. Robert Flaherty avait déjà mélangé plans « sur le vif » et plans répétés dans un autre espace-temps, fondus sans que le spectateur y prenne garde par la grâce 
des raccords-objet, des raccords-lumière, des raccords-mouvement et de l'étalonnage, pour son Nanouk l'Eskimau (Nanook of the North, 1922).

20. Style de filmage imitant les prises de vue d'opérateurs d'actualité pris dans le feu de l'action et par là même peu soucieux de fini plastique ; voir Jullier (2013) : <http://map.revues.org/1371>.

21. Le néoformalisme est entendu ici comme l'imposition faite au contenu de dicter la forme ; le terme de "profilmique» désigne, lui, dans la tradition de l'Institut de filmologie français, l'ensemble des objets réels qui se trouvent devant la caméra, imprimant leur trace sur la pellicule.

22. Sans parler du développement d'une foule de dispositifs de connexion kinesthésique entre le visuel et les mouvements du corps : voir, à propos de l'arrivée des travellings avant dans la vie quotidienne, Jullier (2012).

\section{RÉSUMÉS}

Dans le champ de l'histoire des styles cinématographiques, une lecture courante consiste à dire que la manière de filmer de Leni Riefenstahl, au moins telle que la postérité l'a fixée dans Le Triomphe de la volonté et Les Dieux du stade, annonce ou préfigure le style postmoderne. L'article discute cette affirmation d'une « contemporanéité rétrospective » de deux manières : d'une part en montrant que les inventions stylistiques de Leni Riefenstahl sont davantage des systématisations de trouvailles empruntées aux figures du Zeitgeist; d'autre part en suggérant que d'une manière générale une figure de style qui est techniquement la même, à cinquante ans de distance, n'est plus appréhendée de la même façon quand l'environnement sensoriel de l'époque a changé.

To see Leni Riefenstahl's film style, especially with Triumph of the Will and Olympia, as a foreshadowing of the postmodern way to shoot and edit movies, now has became a platitude in the history of film style. This essays intends to discuss the legitimacy of this aesthetic filiation or, to put it in another way, of this "retroactive contemporaneity". Two reasons are put forward to the discussion; first, Leni Riefenstahl so-called formal inventions are more like systematic reenactments of some cinematic figures of her own time than out-of-Zeitgeist creations; then, an aesthetic form may remain exactly the same on a technical point of view fifty years later, and in the same time it appears utterly different when the perceptual background is no longer the same.

\section{INDEX}

Mots-clés : cinéma, histoire des techniques du cinéma, figures de style, histoire du regard Keywords : Cinema, history of cinematic techniques, motion picture terminology, history of seeing 


\section{AUTEUR}

\section{LAURENT JULLIER}

IECA, Université de Lorraine ; IRCAV, Université Sorbonne Nouvelle-Paris 3 